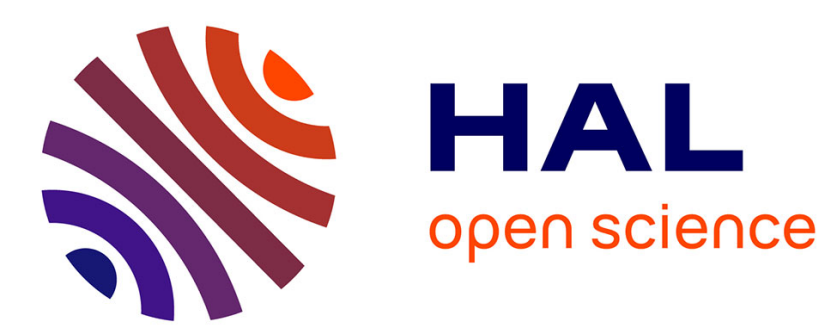

\title{
La passion du vol: de Léonard de Vinci à Jean Letourneur
}

\author{
Bruno Chanetz
}

\section{To cite this version:}

Bruno Chanetz. La passion du vol: de Léonard de Vinci à Jean Letourneur. Arts et sciences , 2020, 1, pp.1-13. 10.21494/ISTE.OP.2020.0466 . hal-02459118

\section{HAL Id: hal-02459118 https://hal.science/hal-02459118}

Submitted on 29 Jan 2020

HAL is a multi-disciplinary open access archive for the deposit and dissemination of scientific research documents, whether they are published or not. The documents may come from teaching and research institutions in France or abroad, or from public or private research centers.
L'archive ouverte pluridisciplinaire HAL, est destinée au dépôt et à la diffusion de documents scientifiques de niveau recherche, publiés ou non, émanant des établissements d'enseignement et de recherche français ou étrangers, des laboratoires publics ou privés. 


\title{
La passion du vol : de Léonard de Vinci à Jean Letourneur
}

\author{
The passion for flight: from Leonardo da Vinci to Jean Letourneur
}

\author{
Bruno Chanetz ${ }^{1}$ \\ ${ }^{1}$ ONERA, président du Haut conseil scientifique de l'Association aéronautique et astronautique de France (3AF)
}

RÉSUMÉ. Léonard de Vinci a embrassé les carrières d'ingénieur et de peintre avec un égal talent et une même réussite. S'il n'est cependant pas l'auteur de la théorie du vol, due quatre siècles plus tard à un autre autodidacte Fréderik Lanchester, il en a posé les premiers jalons. Cet article rappelle sa quête pour arracher l'homme à l'attraction terrestre. II se poursuit par l'évocation de l'œuvre d'un médecin Etienne-Jules Marey, qui au crépuscule du XIXe siècle réalisa les premières images du mouvement fluide. On présente ensuite les visualisations réalisées dans la seconde partie du XXe siècle par un ingénieur de l'ONERA Henri Werlé. Ses films et ses photographies scientifiques furent une source inépuisable d'inspiration de l'artiste Jean Letourneur pour son œuvre sculpté et dessiné, prouvant qu'encore aujourd'hui l'art et la science ne sont pas des univers disjoints.

ABSTRACT. Leonardo da Vinci has embraced the careers of engineer and painter with equal talent and equal success. However, he was not the author of the theory of theft, due four centuries later to another autodidact Frederik Lanchester, he laid the groundwork of this theory. This article recalls his quest to wrest man from earthly attraction. It continues with the evocation of the work of a physician Etienne-Jules Marey, who at twilight of the 19th century produced the first images of the fluid movement. We then present the visualizations made in the second half of the 20th century by an ONERA engineer Henri Werlé. His films and scientific photographs have been an inexhaustible source of inspiration for the artist Jean Letourneur for his sculpted and drawn work, proving that still today art and science are not disjointed universes.

MOTS-CLÉS. théorie du vol, visualisations hydrodynamique, visualisations strioscopiques, sculpture.

KEYWORDS. theory of theft, hydrodynamic visualizations, Schlieren photographs, sculpture.

\section{1) introduction : De la légende à la réalité}

La légende d'Icare et Dédale s'enfuyant du Palais de Minos fait partie des mythes fondateurs de notre civilisation. Elle exprime l'un des rêves les plus vieux de l'humanité : voler comme un oiseau. Léonard de Vinci, Il y a un peu plus de cinq siècles, consacra au vol 400 dessins, dont 150 de machines volantes. Sa quête fut longue et couronnée d'insuccès mais il eut le mérite, au soir de sa vie, de bâtir une théorie du vol, qui même imparfaite, avait le mérite de tenir la route ... des airs.

Quatre siècles plus tard, Etienne-Jules Marey, reprit le flambeau en se posant les mêmes questions que Vinci sur le vol des oiseaux et invalidant comme lui, le vol battu. Ce médecin physiologiste réalisa les premières visualisations fluides. Sa machine à fumée, une soufflerie dotée d'un dispositif permettant de visualiser les images du courant d'air autour d'obstacles fut reproduite en 1999 par le Centre national de la cinématographie et exposée lors d'une exposition au musée d'Orsay en 2004. Cette exposition présentait les superbes clichés réalisés par Marey cent ans auparavant, à l'époque où décollait enfin le plus lourd que l'air.

Cinquante ans plus tard, un ingénieur Henri Werlé débuta une carrière qui devait le conduire à réaliser de magnifiques images scientifiques aux couleurs éclatantes au moyen du tunnel hydrodynamique de l'ONERA à Chatillon-sous-Bagneux.

Ces images présentées en 1973 lors de l'exposition Sciences, Formes, Couleurs au Palais de la Découverte, décidèrent d'une vocation : celle de Jean Letourneur de consacrer sa vie à l'expression du mouvement fluide dans le marbre et le bronze. 


\section{2) Léonard de Vinci et les prémices de la théorie du vol}

\section{Premier ingénieur du roi François ler}

Bien qu'il s'agisse d'un plaidoyer pro domo, mais c'est la loi du genre dans un curriculum vitae, les talents multiples de Léonard de Vinci sont fort bien récapitulés dans son courrier d'offres de service qu'il adresse en 1482 à Ludovic Sforza, duc de Milan. Après avoir détaillé ses compétences dans les domaines du génie civil et de l'ingénierie militaire, il ajoute : je puis exécuter de la sculpture en marbre, bronze ou terre cuite; de même en peinture mon ouvre peut égaler celle de n'importe qui [1] Mieux qu'à Milan, c'est cependant en France où il s'installe au soir de son existence que le génie de Léonard de Vinci fut reconnu à sa plus juste valeur. En 1516, François Ier lui confère le titre de premier peintre, premier ingénieur et premier architecte du roi et met à sa disposition le château de Cloux. Devenu le Clos Lucé, ce manoir perpétue la mémoire de son glorieux locataire.

Si la notoriété de Léonard de Vinci comme ingénieur est bien établie, elle est tardive et ses réflexions dans le domaine du vol furent inconnues pendant plusieurs siècles. Aussi 50 ans après Léonard de Vinci, Benedetto Castelli énonce que dans un canal, la vitesse et la section varient en sens inverse. Ce principe, capital pour la mécanique des fluides, avait pourtant été découvert par Vinci. Malheureusement le seul traité publié tout de suite après la mort de Vinci par son disciple Melzi était consacré uniquement à la peinture : le codex Urbinas.

C'est seulement en 1796 qu'arrivent en France les premiers dessins de Vinci consacrés au vol. Une partie d'entre eux est conservée depuis à la bibliothèque de l'Institut de France. Ces ressources documentaires de premier plan sont le fruit du pillage de l'Italie par les armées de Bonaparte au cours de la première campagne d'Italie (1796-1797).

A la fin du XIXe siècle, les premiers dessins originaux de Vinci sont publiés par Abel Hureau de Villeneuve dans le mensuel l'Aéronaute [2]. En 1893, parait le codex sur le vol des oiseaux : codice sul volo degli ucelli.

\section{L’hélice volante}

En 1881 Gilberto Govi (1826-1889) présente à l'Académie des sciences un mémoire sur l'hélice aérienne de Vinci [3]. Cette hélice, qu'on s'accorde à présenter comme l'ancêtre de l'hélicoptère, est présentée en trois dimensions dans le jardin entourant la demeure du Clos-Lucé que la famille SaintBris a transformé en «parc d'attraction ». Elle figure parmi les machines les plus emblématiques rêvées par Léonard de Vinci.

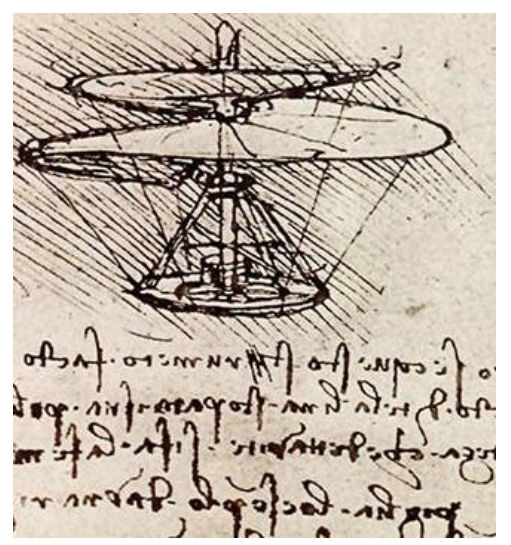

Dessin de Léonard de Vinci

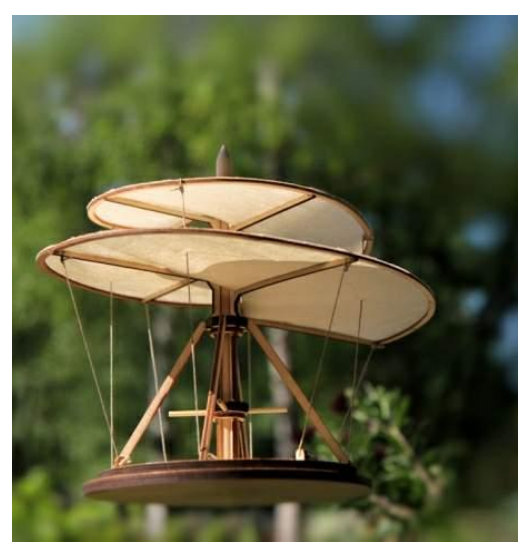

sa réalisation au Clos-Lucé

Crédit Bibliothèque de l'Institut de France

(manuscrit B, fol. 83v-88r) 
Pour autant le principe de cette vis n'est pas dû à Léonard de Vinci. L'idée est reprise du traité $D e$ ingeneis de l'ingénieur siennois Mariano di Jacopa, alias Taccola (1382-1458), dit 1'Archimède de Sienne [4]. Chez Taccola la vis volante est décrite comme ludus puerorum, jouet pour enfant. D'ailleurs un tableau de 1460, dû au Maître de Vivoin, exposé au musée de Tessé (Le Mans), montre un enfant tenant une coquille de noix creuse traversée d'un axe et surmonté d'une hélice [3]. En tirant sur la ficelle, l'hélice tourne. Est-ce un hélicoptère jouet ou un simple moulinet ?

La littérature de la Renaissance, se fait également l'écho d'un tel jouet. Rabelais conte dans Gargantua (1534) : Et pour s'ébattre comme les petits enfants du pays, lui firent un beau virolet des ailes d'un moulin à vent $d u$ Mirebalais [3]. Mirebeau est une petite ville de la Vienne entre Loudun et Poitiers, territoire subissant aujourd'hui les assauts répétés des promoteurs éoliens, attirés un peu par le potentiel vent de la région ... et plus encore par les prébendes accordées par l'Etat.

En remontant plus avant dans le temps, on apprend que ce moulinet existait déjà au $4^{\mathrm{e}}$ siècle en Chine, Ko Hung (283-343) révélant l'existence de jouets en bois constitués d'une planchette vrillée qui bondit en l'air lorsqu'on fait tourner l'axe [3].

Entre 1486 et 1496, Léonard de Vinci écrit : Si cet instrument en forme de vis est bien fait-c'est à dire fait en toile de lin cerclé dont les pores auront été obturés avec de l'empois - et proprement tourné, cette hélice tracera sa spirale en l'air et il montera haut [3].

La vis de Léonard de Vinci pèse un poids énorme avec son diamètre de $10 \mathrm{~m}$. Face à cette masse, la puissance musculaire de l'homme est impuissante. Pourtant 20 ans après ses premières études, Léonard de Vinci refuse d'accepter l'insuffisance des performances physiques dans le cas d'un vol mû par la force musculaire [4].

L'acte de naissance de l'hélicoptère date du $1^{\text {er }}$ mai 1784 , où un rapport de l'Académie des Sciences exprime ses conclusions après avoir examiné un modèle de $85 \mathrm{~g}$ 《simple et ingénieux » élaboré par les inventeurs Launoy et Bienvenu. Deux paires d'hélices tournant en sens contraire, ces ailes étant disposées de manière que les percussions horizontales de l'air se détruisent et que les percussions verticales conspirent à élever le moteur [3].

\section{Vol à ailes battantes}

Entre 1485 et 1490, Léonard de Vinci effectue ses premières études sur le vol avec transmission de force directe du pilote sur la machine. Entre 1490 et 1500, il conclut à l'impossibilité du vol musculaire, mais croit encore à celui du vol instrumental : poulie, ressort, manivelle, pédale ...

Or le principe de l'aile battante, imitée des insectes et des oiseaux ne fonctionnent pas pour des gros aéronefs. Actuellement des micro drones, tel le projet Remanta [5] de l'ONERA qui imite la libellule, fonctionnent sur ce principe, mais justement parce qu'il s'agit d'objets volants très petits, de la taille du pouce.

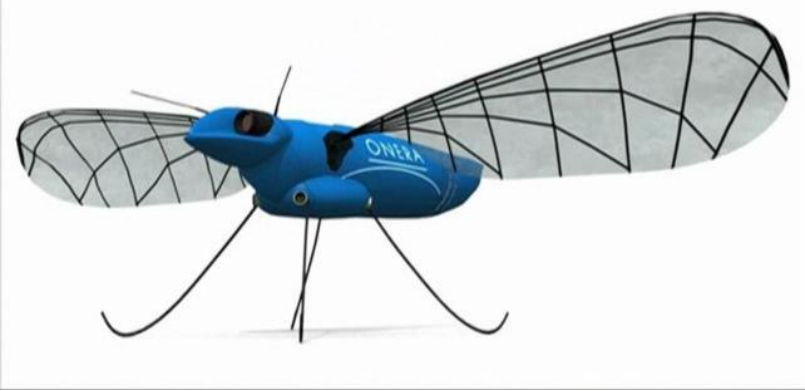

Micro drone Remanta (crédit ONERA) 
Le mathématicien Jean-Baptiste Dante construisit des ailes artificielles permettant à l'homme de s'élever dans les airs et fit plusieurs essais de son appareil sur le lac de Trasimène, dont la tradition ne rapporte pas des succès vibrants. Pourtant dans une fête donnée à l'occasion du mariage de Bartoloméo Alviano, il semble que Dante offrît un spectacle inédit à la ville de Pérouse. Au moyen de son appareil, il se serait élevé très haut au-dessus de la place. Mais, le fer avec lequel il dirigeait l'une de ses ailes s'étant brisé, il tomba sur l'église Notre-Dame et se cassa une cuisse. [2].

Léonard de Vinci aurait produit un ou plusieurs aéronefs pour Gian Antonio Di Mariolo. Il note en avril 1505 : De la montagne qui porte son nom, le fameux oiseau prendra son essor, qui de sa grande renommée emplira le monde. Jérôme Cardan, dans ses mémoires confirme qu'une tentative de vol aurait eu lieu, sur les flancs du mont Ceccri (Cercero) au-dessus de Fiesole, sans doute un planeur battant des ailes, mais ce fut un échec [3].

Cependant Léonard de Vinci reste convaincu que l'homme pourra dominer l'air et s'élever audessus de celui-ci grâce à l'invention de grandes ailes qui opposeront une résistance à cet élément récalcitrant et le soumettront [4]. Ces dessins des ailes de chauves-souris inspireront quatre siècles plus tard Clément Ader, qui fit en 1890, soit treize ans avant Orville et Wilbur Wright, le premier saut de puce avec un plus lourd que l'air motorisé, vol malheureusement non homologué.

\section{Pourquoi I'homme ne vole pas comme un oiseau?}

En effet ce qui manquait à Léonard de Vinci, c'était un moteur. Peut-être avait-il remarqué que tous les oiseaux ne volent pas ? L'autruche, qui atteint $150 \mathrm{~kg}$, ne vole pas. Le manchot empereur d'une masse de $40 \mathrm{~kg}$ ne quitte pas le sol. L'outarde Kori, avec une masse de $20 \mathrm{~kg}$, est le plus grand animal volant, mais elle doit prendre son envol d'un point haut. De même le cygne doit acquérir une grande vitesse avant que sa portance soit supérieure à son poids. Inversement les oiseaux de faible charge alaire décollent avec une extrême rapidité, tels les moineaux. La charge alaire exprime le poids supporté par chaque mètre carré d'aile en $\mathrm{N} / \mathrm{m}^{2}$ : la mouche drosophile affiche $3,5 \mathrm{~N} / \mathrm{m}^{2}$, la libellule $5 \mathrm{~N} / \mathrm{m}^{2}$, l'oiseau-mouche, d'une masse de $1,5 \mathrm{~g}$, a une charge alaire de $20 \mathrm{~N} / \mathrm{m}^{2}$ et le pigeon de $30 \mathrm{~N} / \mathrm{m}^{2}$. Quant à la cigogne, elle atteint $66 \mathrm{~N} / \mathrm{m}^{2}$ et l'albatros $140 \mathrm{~N} / \mathrm{m}^{2}$ [6]. On se souvient de la scène mémorable du dessin animé de Walt Disney, où Orville, pilote d'Albatross Air Service dont le nom est un clin d'œil aux frères Wright, peine à décoller avec ses passagers Bernard et Bianca.

Pour équilibrer le poids, la portance doit être telle que la vitesse soit de l'ordre de grandeur du poids à la puissance 1/6. Ainsi plus la charge alaire est importante, plus l'animal est contraint de voler vite : Le Boeing 747 (400 tonnes) volent à $900 \mathrm{~km} / \mathrm{h}$, soit dix fois plus vite que le faucon (1 $\mathrm{kg}$ ) qui vole à $45 \mathrm{~km} / \mathrm{h}$, soit dix fois plus vite que la mouche qui vole à $6 \mathrm{~km} / \mathrm{h}$. L'homme devrait voler à $100 \mathrm{~km} / \mathrm{h}[6]$.

Quatre siècles plus tard, Etienne-Jules Marey établit que la puissance musculaire de l'homme devrait être 200 fois plus grande pour qu'il puisse s'élever en actionnant des ailes artificielles. JeanMarc Jancovici, professeur à l'Ecole des Mines, faisait dernièrement cette comparaison évocatrice : la puissance développée par un simple robot ménager équivaut à celle de quatre cyclistes [7].

Prenant pour référence l'oiseau colibri, le plus lourd des animaux à sang chaud capable de voler sur place en l'absence de vent, Jean-Michel Courty et Edouard Kierlik, ont établi qu'un homme de $75 \mathrm{~kg}$ recouvrant ses bras de plumes aurait besoin de $7000 \mathrm{~W}$ pour pratiquer le vol stationnaire, alors qu'un sportif développe seulement en effort prolongé une puissance de 500 Watt [3]. Il faut bien en convenir nous sommes beaucoup trop lourds et trop faibles pour voler. 


\section{Les prémices de la théorie du vol de Léonard de Vinci}

Après 1505, Léonard de Vinci abandonne l'idée des ailes battantes et développe des idées sur la force ascensionnelle (portance). Il réfléchit à des appareils à voilure fixe, définit le vol plané. L'air qui s'écoule autour de l'oiseau exerce sur lui des forces. L'air s'oppose à son avancement. C'est la traînée, qu'il lui faut vaincre pour avancer. L'air, en mouvement autour des ailes, le porte. C'est la portance, qui s'oppose au poids et le maintient en l'air.

Il en déduit fort justement l'importance de soigner l'arrière du profil pour réduire la traînée et postule deux lois relatives à la traînée [8] :

- elle serait proportionnelle à la surface des ailes ce qui est vrai,

- elle serait proportionnelle à la vitesse, ce qui est faux, puisque c'est au carré de la vitesse.

C'est dire que la vitesse est capitale pour voler et le capitaine Ferdinand Ferber (1862-1909), le premier à avoir réussi en 1905 pour l'Europe l'homologation d'envol d'un avion motorisé, deux ans après les frères Wright, exprime joliment cette vérité : La portance est une fleur qui nait de la vitesse.

\section{3) Les pionniers de l'air et les premières visualisations du mouvement fluide}

\section{Clément Ader (1841-1925), lointain successeur de Léonard de Vinci invente l'avion}

En s'inspirant de la chauve-souris, Clément Ader réalise un véhicule à moteur capable de s'élever un peu dans les airs et de se reposer. Le 9 octobre 1890 à Arminvilliers, à bord d'Eole, il parcourt une cinquantaine de mètres à une vingtaine de centimètres seulement au-dessus du sol. Cet appareil de $300 \mathrm{~kg}$ était équipé d'un moteur à vapeur de 20 chevaux. C'est le premier à avoir décollé, mais si peu ... Avec un moteur moins lourd, il eut peut-être réussi à s'élever un peu plus haut. Si la paternité du premier vol à moteur lui est disputée, en revanche Clément Ader inventa un mot destiné à l'immortalité : avion [9].
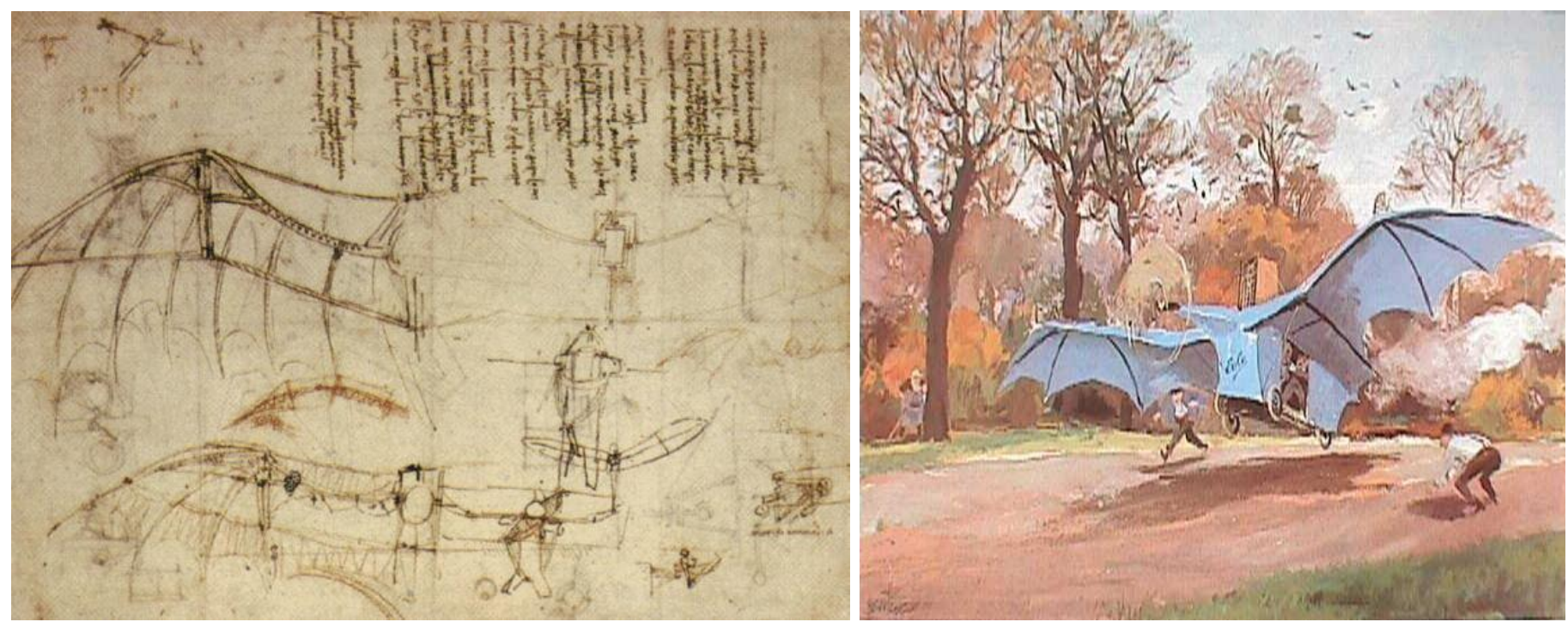

Dessin de Léonard de Vinci d'ailes de chauve-souris et l'Éole de Clément Ader en 1890 Crédit Bibliothèque de l'Institut de France (manuscrit B, fol. 83v-88r)

\section{Le roi pétrole préside à l'essor du vol}

Plus que toute autre technologie, l'avion doit son existence à la transition énergétique. J'évoque bien sûr la transition énergétique ... qui au cours du XIXe siècle nous a fait basculer dans l'ère 
moderne. Délaissant nos moulins à vents dont la production d'énergie intermittente ne permettait pas l'émergence d'une société industrielle, nous nous sommes tournés vers le charbon, puis le pétrole, qui représentaient un potentiel énergétique combien plus efficace et plus facilement mobilisable. Sans le moteur à explosion l'avion n'aurait pas existé.

\section{De Magnus à Lanchester : la difficile gestation de la théorie du vol}

En 1852, un Prussien Gustav Magnus, s'intéressa à l'effet de giration des boulets de canons, phénomène qu'on rencontre aussi lorsque les balles ou ballons ont de 1' «effet ». Il établit ainsi que la rotation d'un boulet infléchissait la trajectoire balistique avec pour conséquence le ratage de la cible. La force de Magnus - ou effet Magnus - n'est autre qu'une force de portance ou de déportance selon le sens de giration. Tout était dès lors en place - ou presque - au niveau des éléments théoriques, pour permettre d'appréhender le vol [10]

En 1894, quelques années avant les travaux de Marey et neuf ans avant le vol des frères Wright, Lanchester énonça une théorie du vol, dite théorie de la circulation. Il l'éprouva sur des modèles réduits. En 1907, il publia Aerial Flight, ouvrage qui définissait les forces de portance et de traînée, qui eut un écho en Allemagne, où Ludwig Prandtl établit mathématiquement la théorie de Lanchester.

Il fallut en effet attendre la charnière du $\mathrm{XIX}^{\mathrm{e}}$ et du $\mathrm{XX}^{\mathrm{e}}$ siècle, quand l'invention du moteur thermique mit à disposition des pionniers de l'air une propulsion suffisante, pour que ceux-ci élaborent des profils cambrés dont ils allaient équiper leurs aéronefs.

\section{Gustave Eiffel met en évidence la composante aspiration de la force de portance}

On le sait peu, mais Gustave Eiffel apporta une contribution essentielle à l'aérodynamique expérimentale en construisant une soufflerie [11] aux pieds de la Tour éponyme, qu'il transféra en 1912, rue Boileau à Auteuil, où elle fonctionne encore. Ces essais lui permirent de mettre en évidence l'aspiration qui contribue essentiellement à la force de portance [12] :

Cette étude a fait ressortir l'importance prépondérante des dépressions à l'arrière et a montré que l'aile de l'aéroplane est deux fois plus aspirée par l'air qui s'écoule sur sa face dorsale, qui n'est poussée par l'air qui s'écoule sur sa face ventrale. Avant que ce fait ne fût mis en évidence au Laboratoire du Champ de Mars, les constructeurs d'avions ne tenaient pas compte des dépressions sur la face dorsale pour l'attache des toiles des ailes, et cela a dî amener des catastrophes par déchirure inexpliquées de cette toile pendant le vol. On y a remédié depuis la publication de mes travaux.

\section{Etienne-Jules Marey réalise les premières images du fluide en mouvement}

Marey a produit dans un espace clos à parois transparentes un courant d'air régulier en faisant arriver dans ce courant d'air des filets de fumée parallèles et équidistants. Il place à la rencontre de ces filets des surfaces de formes diverses sur lesquelles ils s'infléchissent diversement. Marey: Eclairer vivement ces fumées et en photographier instantanément l'apparence, tel était le programme à remplir, ce qu'il fit de 1899 à 1901 [13]. 


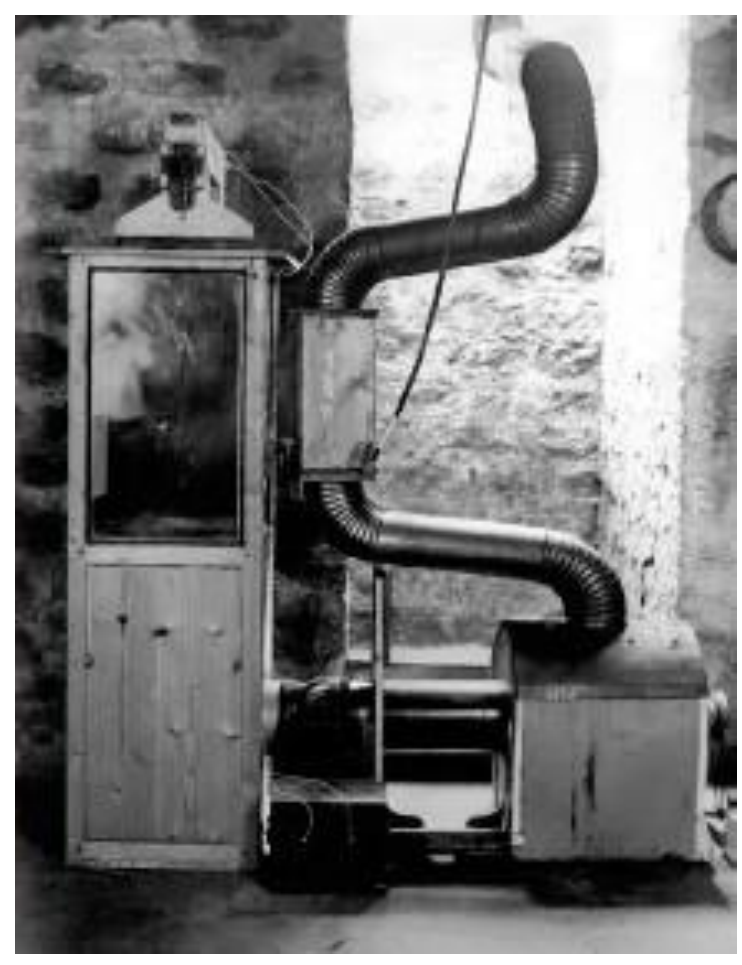

La soufflerie à fumée de Marey

La fumée est produite dans une boite métallique par la combustion d'étoffes de coton et d'amadou. Les filets se forment en passant par une rampe constituée de tubes d'émission en plomb. Ils sont aspirés vers le bas grâce à un ventilateur électrique et prennent alors l'apparence de cordes tendues comme celles d'une harpe. Les clichés représentent le comportement des filets de fumée lorsqu'ils rencontrent un plan incliné selon des angles variés. Ils sont réalisés par un appareil photographique placé devant la vitre de la machine accompagnée d'un flash à éclair magnétique.

La dernière version de la machine de Marey indique la vitesse de l'air. Un trembleur électrique une simple sonnette sans timbre - ébranle dix fois par seconde les petits tubes qui conduisent la fumée : les filets véhiculent des ondes successives dont l'écartement correspond à l'espace parcouru par l'air à chaque dixième de seconde.
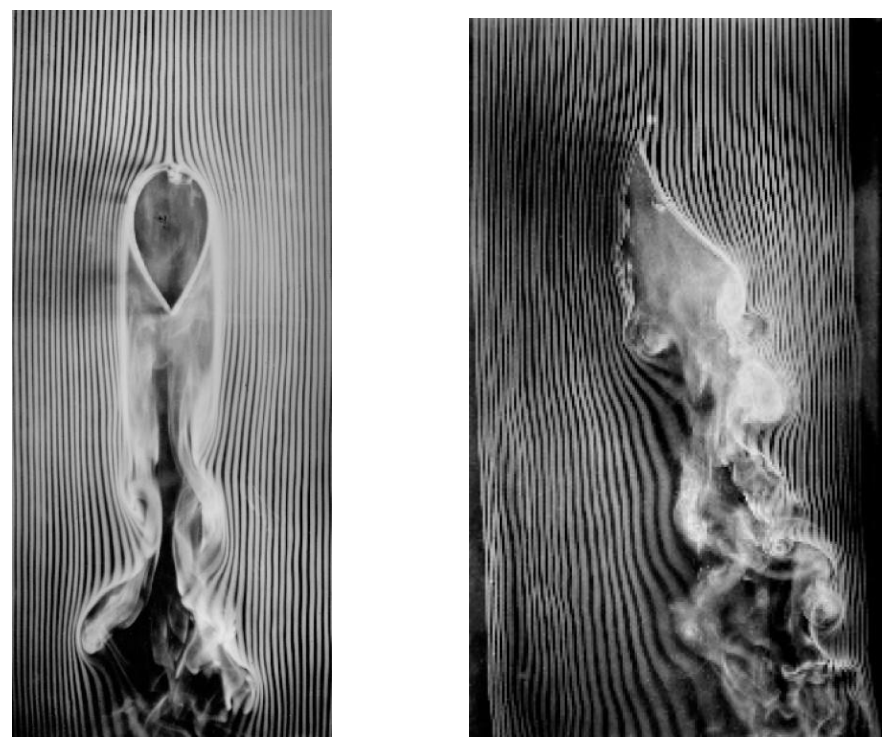

Visualisations réalisées par Marey (crédit Cinémathèque française) 
Mais Marey renonce à poursuivre ces expériences: Mes connaissances insuffisantes en mathématiques ne me permettent pas de quitter le terrain expérimental. Je suis même parfois bien embarrassé pour interpréter certains résultats d'expériences, par exemple les trajets bizarres que suivent les filets de fumée.

Marey était un modeste, qualité qui avait été épargnée à Léonard de Vinci, lequel n'avait jamais éprouvé un sentiment d'infériorité, malgré de criantes lacunes en mathématiques, concernant par exemple le calcul des fractions ou la détermination des racines carrées ...

\section{4) D’Henri Werlé à Jean Letourneur, le sculpteur du mouvement fluide}

\section{Henri Werlé ou « le maître » du tunnel hydrodynamique de l'ONERA}

Les images de Werlé se situaient en droite ligne des études de Marey. Ingénieur à l'ONERA, il fut actif durant la seconde moitié du XXe siècle. Il réalisa pendant sa vie professionnelle des milliers de clichés d'écoulements au tunnel hydrodynamique, à l'origine d'un apport important à l'aéronautique et source inépuisable d'inspiration artistique [14].

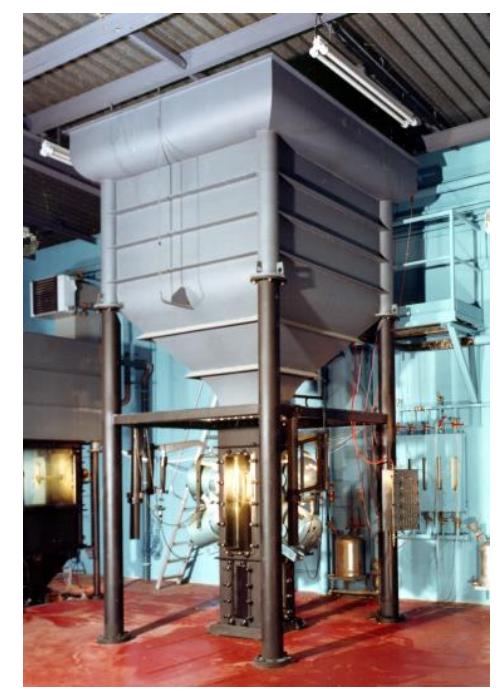

Le tunnel hydrodynamique de L'ONERA à Châtillon (Hauts-de-Seine)

Parmi les visualisations réalisées par Werlé dans les années 60, certaines ont fait le tour du monde. Ainsi les célèbres photos représentant les enroulements tourbillonnaires sur une maquette de Concorde.
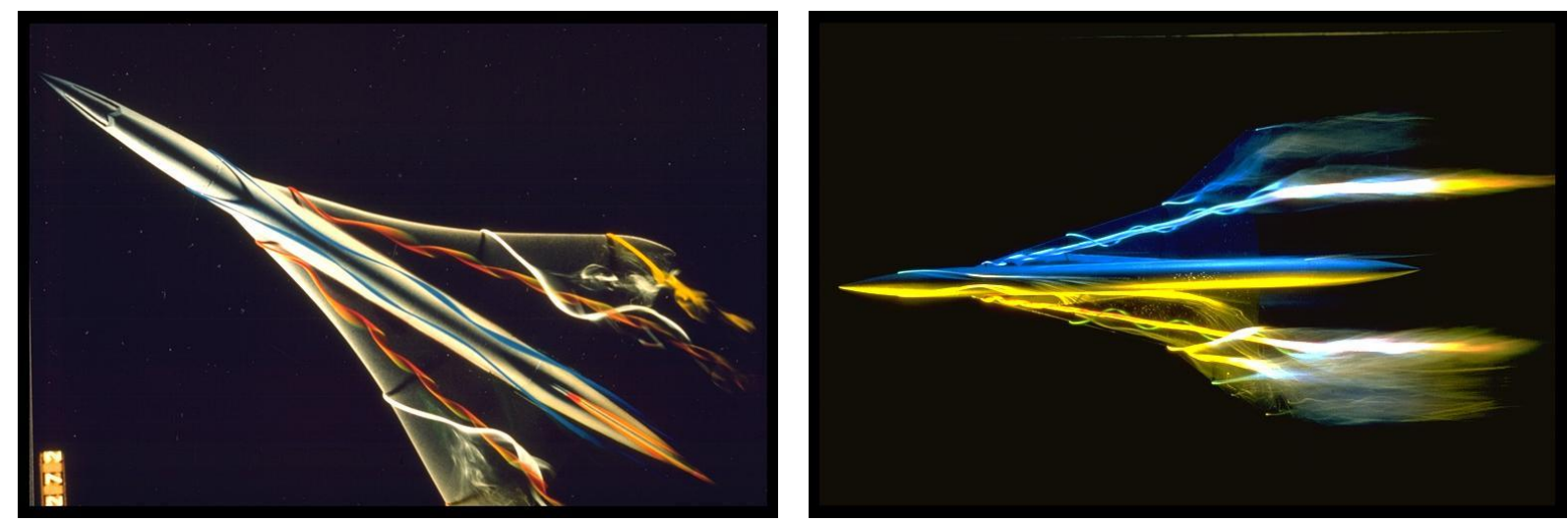

Visualisations des enroulements tourbillonnaires sur Concorde (crédit ONERA) 
Aussi populaire que Concorde, la fameuse DS de Citroën a également été testée au tunnel hydrodynamique. On note le décollement de la Couche limite qui se produit au milieu du toit de la DS, produisant un important sillage, source principale de la trainée du véhicule.

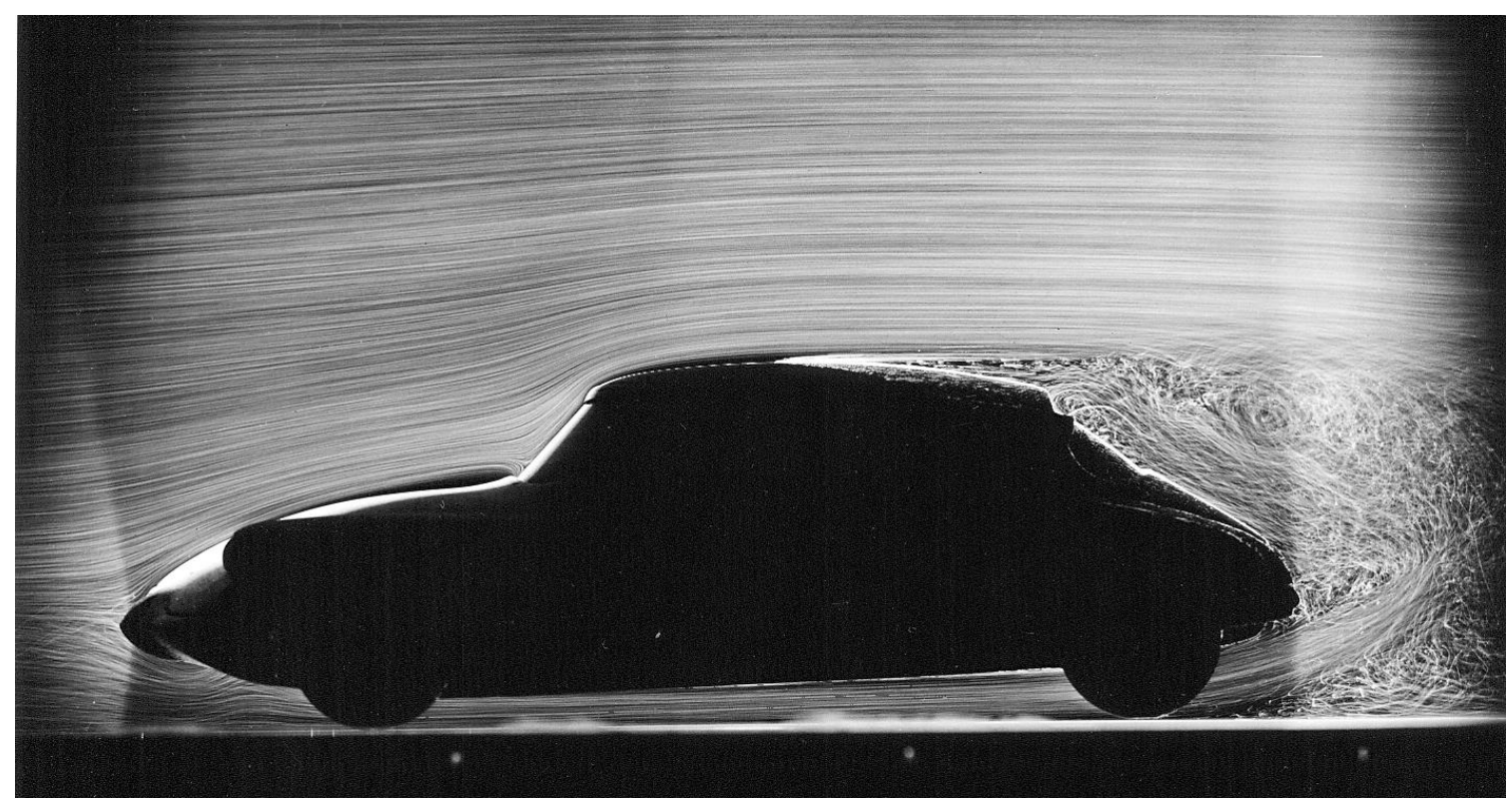

Visualisation au tunnel hydrodynamique autour d'une DS Citroën (crédit ONERA)

\section{Jean Letourneur, interprète de l'instantané}

«J'aurai vécu avec Henri Werlé qui prit sa retraite en 1988 la fin d'un cycle initié au début du XVIe siècle avec Léonard de Vinci » déclare Jean Letourneur, professeur agrégé, enseignant dans différentes écoles d'art parisiennes jusqu'en 2019. C'est un passionné d'aérodynamique. Sa vocation de sculpteur il l'a trouvée dans son berceau, auprès de son père René Letourneur, Premier Grand Prix de Rome de sculpture en 1926. Sa passion il l'exerce aujourd'hui encore dans l'atelier paternel à Fontenay aux Roses. C'est auprès de lui, dont il fut le praticien durant 8 ans, qu'il acquiert la technique de la taille directe. Il est à l'heure actuelle l'un des rares sculpteurs français à maîtriser cette technique qui ne tolère pas la faute. Comme il le souligne : Tailler le marbre ne s'improvise pas, l'erreur est interdite. Son attrait pour les sciences il le doit également au milieu familial, son grand-père maternel Henri Gondet ayant dirigé durant trente ans les Etablissements Beaudoin (fabricants d'instruments de mesures de précision) avant de devenir Directeur Scientifique de la Physique à l'ONERA puis de prendre la direction des laboratoires du CNRS de Bellevue à Meudon, à la demande de Frédéric Joliot-Curie. Jean Letourneur a trouvé son chemin de Damas en 1973, l'année de son bac, en visitant l'exposition Sciences, Formes, Couleurs au Palais de la Découverte, où l'ONERA présentait ses travaux en Mécanique des Fluides. Ce fut pour lui la révélation qui allait décider de sa carrière.

Cette carrière est le fruit de sa rencontre avec Henri Werlé, maître de recherche à l'ONERA, dont les travaux en hydrodynamique l'ont littéralement subjugué. Les très belles visualisations réalisées avec des colorants au tunnel hydrodynamique par Henri Werlé ont fait les heures de gloire de l'ONERA. Elles ont fasciné Jean Letourneur, qui a vu prendre vie aux images fixes de l'exposition du Palais de la Découverte. Il lui suffisait de regarder et de dessiner, puis de sculpter.

À cette époque, le calcul numérique était encore impuissant à modéliser finement les écoulements aérodynamiques. Aussi les études expérimentales étaient nombreuses et parmi elles, les procédures initiées par Marey, transposées dans l'eau avec des colorants. Jean Letourneur rapprocha 
immédiatement ces études des dessins de Léonard de Vinci, fondateur de cette science et de la série des Déluges (voir article suivant).

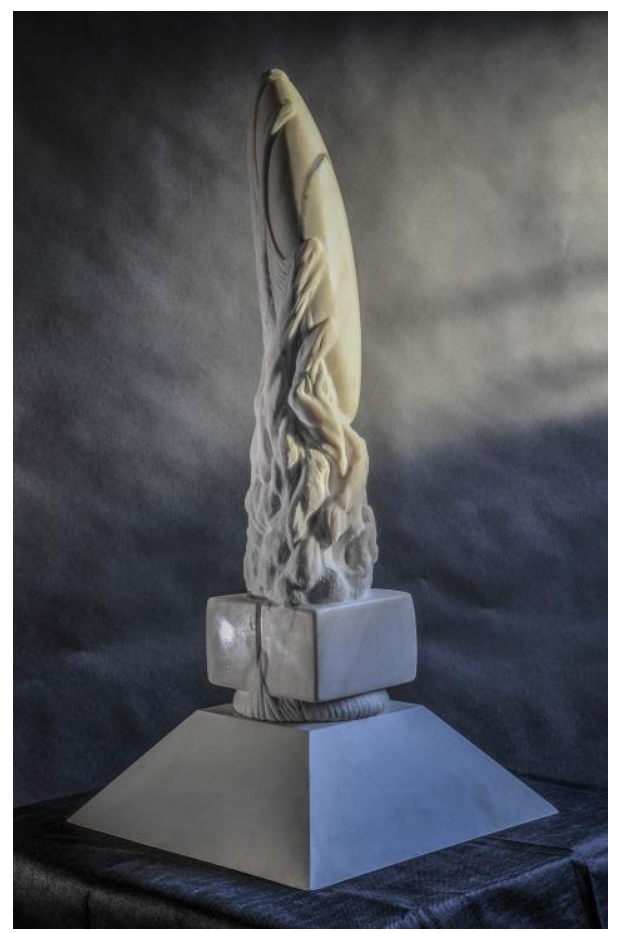

Le Miroir 1994, Marbre, $100 \times 49 \times 49 \mathrm{~cm}$

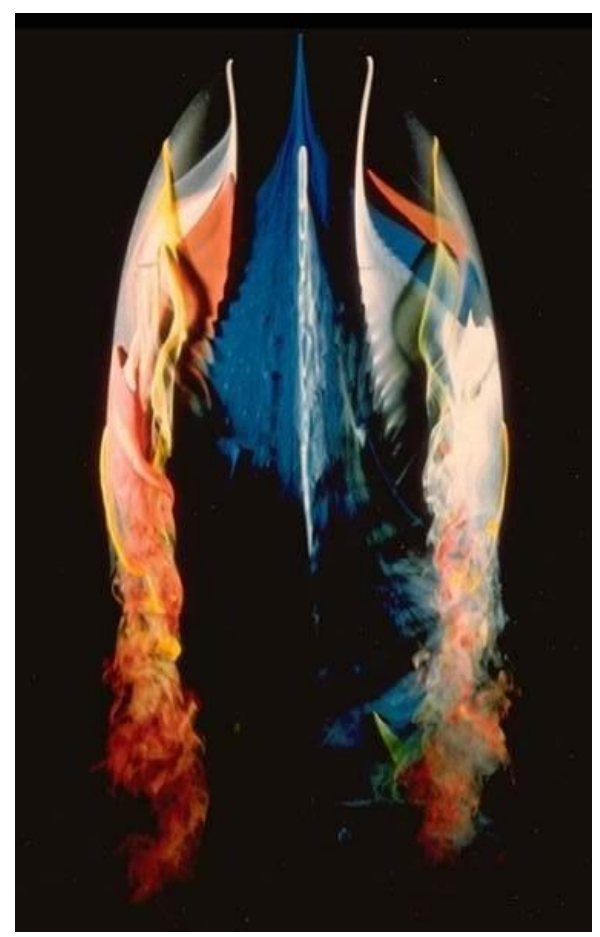

visualisation d'un ellipsoïde en incidence (Cliché Werlé crédit ONERA)

Parmi les œuvres issues de cette fructueuse collaboration entre l'artiste et le scientifique, je n'en citerai qu'une qu'y m'est chère : Le Miroir (1994). Cette œuvre, présentant un ellipsoïde, évoque les miroirs en bronze poli de l'antiquité, dont elle revêt la matière. Le socle, constitué de filaments fluides entrelacés exprime avec vigueur les enroulements tourbillonnaires qui prennent naissance à l'extrados de l'ellipsoïde en incidence. Magnifique alliance du passé et du présent et symbiose entre l'art et la science qu'aurait appréciées Henri Poincaré, l'étude révélant aussi l'existence des points singuliers, révélés par ce grand mathématicien cent ans auparavant [15]. Les recherches de Werlé constituaient les prémices de la thèse que je débutai en 1983 à l'ONERA [16]. Cette sculpture est un double hommage rendu à Jean Letourneur, qui a si remarquablement magnifié le mouvement fluide, et à Henri Werlé, dont les superbes clichés ont fait les heures de gloire de l'ONERA. Et ce n'est pas le moindre mérite d'Henri Werlé d'avoir, en servant de mentor à Jean Letourneur, suscité une production artistique aussi puissante et originale.

\section{À l'orée du XXIe siècle, Jean Letourneur prend de la vitesse}

En 2005 dans le cadre de l'année internationale de la physique que Jean Letourneur décida de monter en vitesse ! Jusque-là ses sources d'inspirations s'étaient limitées aux écoulements basse vitesse. Il a frappé de nouveau à la porte de l'ONERA. Henri Werlé n'était plus là et j'ai eu le plaisir de faire sa connaissance et de guider ses pas vers le supersonique. Il est venu plusieurs fois au centre de Meudon pour découvrir les souffleries et s'intéresser aux écoulements à grande vitesse. Il a été particulièrement intéressé par la simulation des écoulements hypersoniques dans les souffleries à rafales où des vitesses de $1,5 \mathrm{~km} / \mathrm{s}$ sont atteintes Nous lui avons montré les ondes de choc visualisées au moyen de la méthode strioscopique. Le résultat de cette découverte du domaine des très hautes vitesses fut la création d'une grande médaille pivotante Interférence de chocs, représentant sur une face un croisement de choc du premier type, ou interférence régulière, et sur l'autre face un croisement du second type, ou phénomène de Mach [17]. Cette médaille, orientée de 
bas en haut, rappelle l'envol des fusées, qui s'arrachent à l'attraction terrestre. Quant aux vues de l'écoulement en soufflerie, elles ont une orientation horizontale, le vent venant de la gauche à une vitesse égale à dix fois la vitesse du son, soit Mach 10.
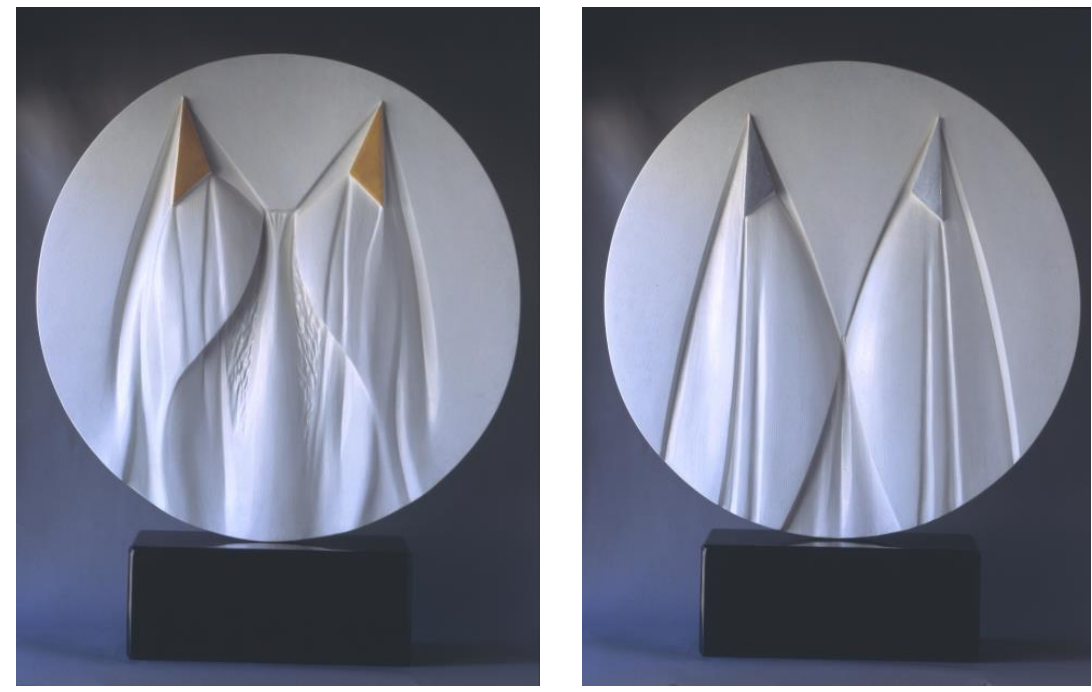

Interférences de chocs, 2005, Stuc, 61 x 7 cm, Année Internationale de la Physique
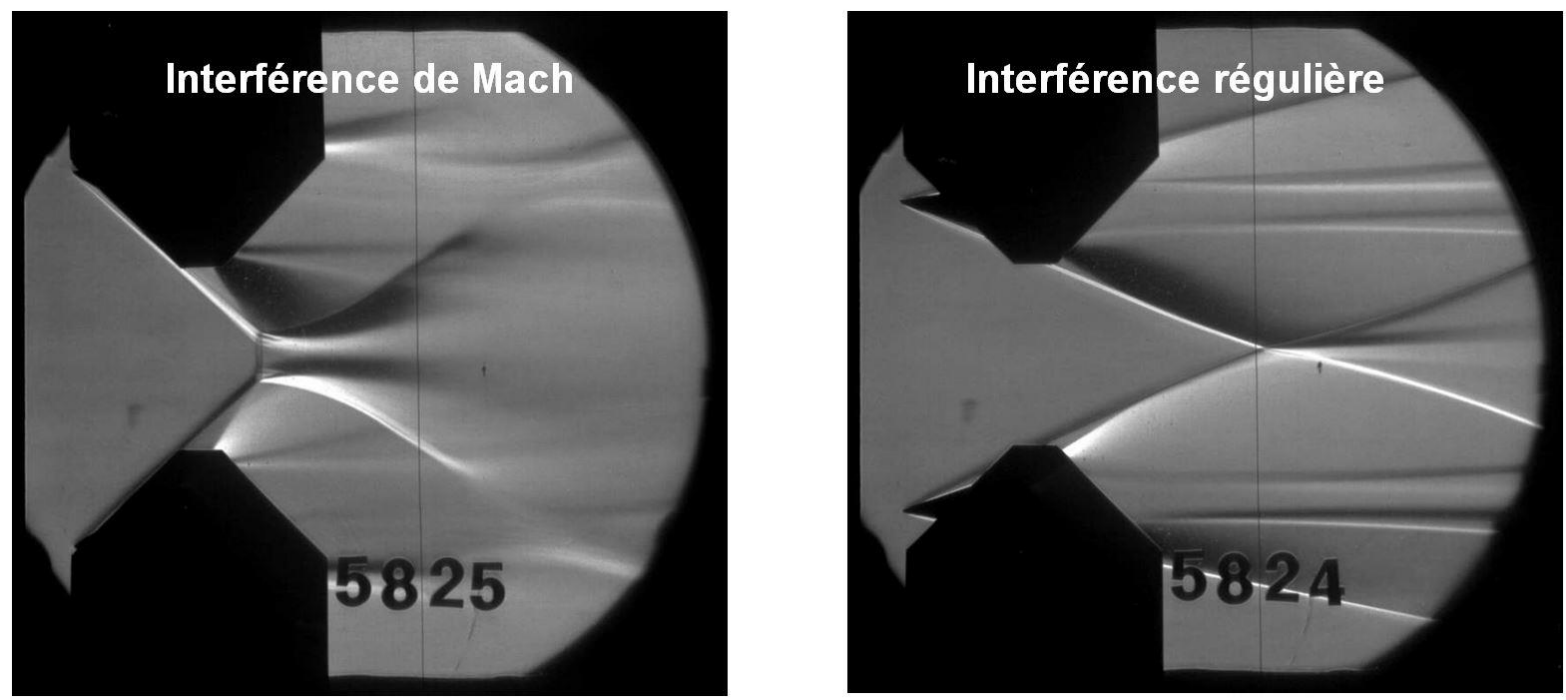

Visualisations strioscopiques réalisées à la soufflerie R3Ch de l'ONERA à Meudon à gauche phénomène de Mach, à droite interférence régulière (Mach 10, soit 1,5 km/s)

Sur la figure de gauche, à Mach 10 à l'aval de deux triangles mobiles, ces interférences donnent naissance par une variation de leur écartement à un « disque de Mach » perpendiculaire à l'écoulement et le ralentissant. Ce disque de Mach est le siège d'un intéressant phénomène d'hystérésis - persistance d'un phénomène quand cesse la cause l'ayant produit - provoqué par la rotation des triangles générateurs (argent et or sur la sculpture). On constate alors l'apparition le long de deux lignes de glissement, des phénomènes turbulents analogues à ceux que Jean Letourneur avait déjà̀ exploités pour les très basses vitesses, ce qui n'est pas étonnant car en aval d'un choc droit à fort nombre de Mach, l'écoulement atteint le bas subsonique. La figure de droite est relative à la configuration régulière, qui apparait pour les plus faibles angles des triangles générateurs. 


\section{5) Conclusion}

J'emprunte ma conclusion à Michael Gibson, critique d'art dans l'International Herald-Tribune, qui a très bien exprimé cet art empreint de science et de technique, cette connivence avec la recherche, qui est la marque de Jean Letourneur : Leonardo da Vinci s'est intéressé le premier à cette mécanique des fluides en variant les obstacles de façon expérimentale et en dessinant les résultats obtenus. Letourneur, qui a appris tout jeune le métier de la taille directe, a choisi d'expérimenter ce genre dans la pierre. Il en résulte un étonnant tourbillon figé, une écume baroque qui arrête sous nos yeux la géométrie des vortex.

L'article suivant permettra au lecteur de découvrir l'œuvre complète de Jean Letourneur avec le meilleur des guides : l'artiste lui-même !

\section{Bibliographie}

[1] Serge Legat

Aéroclub de France, Les cent vies de Léonard de Vinci, Clubs et cercles Magazine, automne 2019, nº48

[2] Léon Darsonval

L'Aéronautique dans le département de l'Aube du XVIIIe siècle à nos jours

https://sites.google.com/site/darsonvalleon/page-1k

[3] Bernard Bombeau

L’Hélicoptère, Privat Editions, 2006

[4] Frank Zöllner et Johannes Nathan

Léonard de Vinci l'œuvre graphique, Edition Taschen, 2003

[5] Agnès Luc-Bouhali

Progress of the Remanta project on mav with flapping wings

https://www.onera.fr/sites/default/files/Departements-scientifiques/DCPS/emav-2006-remanta-mav-flapping-wings.pdf

[6] Marielle Vergès et Kamil Fadel

Le vol des oiseaux et des insectes

Découverte, revue du Palais de la découverte n³06, mars 2003, ISSN 1621-0085

[7] Jean-Marc Jancovici

Conférence à Loudun le 22 octobre 2019

https://www.youtube.com/watch?v=Ubx9YbMz8gM

18] Kamil Fadel

Histoire de la théorie de la sustentation, Découverte, revue du Palais de la découverte ${ }^{\circ} 306$, mars 2003, ISSN 16210085

[9] Philippe Jung

Clément Ader, l'inventeur de l'avion, Lettre 3AF n³5 (janvier-février 2019)

https://www.3af.fr/article/culture/clement-ader-1841-1925-l-inventeur-de-l-avion

[10] Bruno Chanetz

L'application des nombres complexes au calcul des profils d'aile

Comptes Rendus Mécanique Volume 347, Issue 7, July 2019, Pages 544-549

https://www.sciencedirect.com/science/journal/16310721 
[11] Jean Délery, Bruno Chanetz, Patrick Gilléron, Patrick Gnemmi et Philippe Perrier

Aérodynamique expérimentale, souffleries et méthodes de mesure, Cépaduès Editions, 2017

[12] Gustave Eiffel

Résistance de l'Air, principaux résultats, 1922

[13] Marie-Laure Théodule, Laurent Mannoni, Bruno Chanetz

Marey, précurseur oublié des souffleries, La Recherche, n 380, Novembre 2004

[14] Henri Werlé

Principaux types de décollement libre observés sur maquettes ellipsoïdales, Note Technique Onera 1985-7

[15] Henri Poincaré

Sur les propriétés des fonctions définies par les équations aux différences partielles, Gauthier-Villars 1879.

[16] Bruno Chanetz

Contribution à l'étude du décollement tridimensionnel en écoulement turbulent incompressible, Note Technique Onera 1988-6

[17] Bruno Chanetz and Richard Benay

Hysteresis phenomena associated with shock waves interference in steady flow, Int. J. of Aerodynamics, Vol 2, Nos. $2 / 3 / 4,2012$ 